\title{
Efficient Feature Extraction for Fast Segmentation of MR Brain Images
}

\author{
László Szilágyi ${ }^{1,2}$, Sándor M. Szilágyi², and Zoltán Benyó1 \\ ${ }^{1}$ Budapest University of Technology and Economics, \\ Dept. of Control Engineering and Information Technology, Budapest, Hungary \\ ${ }^{2}$ Sapientia - Hungarian Science University of Transylvania, \\ Faculty of Technical and Human Science, Târgu-Mureş, Romania \\ lalo@ms.sapientia.ro
}

\begin{abstract}
Automated brain MR image segmentation is a challenging problem and received significant attention lately. Various techniques have been proposed, several improvements have been made to the standard fuzzy c-means (FCM) algorithm, in order to reduce its sensitivity to Gaussian, impulse, and intensity non-uniformity noises. In this paper we present a modified FCM algorithm, which aims at accurate segmentation in case of mixed noises, and performs at a high processing speed. As a first step, a scalar feature value is extracted from the neighborhood of each pixel, using a filtering technique that deals with both spatial and gray level distances. These features are clustered afterwards using the histogram-based approach of the enhanced FCM algorithm. The experiments using 2-D synthetic phantoms and real MR images show, that the proposed method provides better results compared to other reported FCM-based techniques. The produced segmentation and fuzzy membership values can serve as excellent support for level set based cortical surface reconstruction techniques.
\end{abstract}

Keywords: image segmentation, fuzzy c-means algorithm, feature extraction, noise elimination, magnetic resonance imaging.

\section{Introduction}

By definition, image segmentation represents the partitioning of a digital image into non-overlapping, consistent pixel sets, which appear to be homogeneous with respect to some criteria concerning gray level intensity and/or texture.

The fuzzy c-means (FCM) algorithm is one of the most widely studied and applied methods for data clustering, and probably also for brain image segmentation [26]. However, in this latter case, standard FCM is not efficient by itself, as it is unable to deal with that relevant property of images, that neighbor pixels are strongly correlated. Ignoring this specificity leads to strong noise sensitivity and several other imaging artifacts.

Recently, several solutions were given to improve the performance of segmentation. Most of them involve using local spatial information: the own gray 
level of a pixel is not the only information that contributes to its assignment to the chosen cluster. Its neighbors also have their influence while getting a label. Pham and Prince 8 modified the FCM objective function by including a spatial penalty, enabling the iterative algorithm to estimate spatially smooth membership functions. Ahmed et al. 1] introduced a neighborhood averaging additive term into the objective function of FCM, calling the algorithm bias corrected FCM (BCFCM). This approach has its own merits in bias field estimation, but it computes the neighborhood term in every iteration step, giving the algorithm a serious computational load. Moreover, the zero gradient condition at the estimation of the bias term produces a significant amount of misclassifications [10. Chuang et al. [5] proposed averaging the fuzzy membership function values and reassigning them according to a tradeoff between the original and averaged membership values. This approach can produce accurate clustering if the tradeoff is well adjusted empirically, but it is enormously time consuming.

In order to reduce the execution time, Szilágyi et al. 11, and Chen and Zhang [4] proposed to evaluate the neighborhoods of each pixel as a pre-filtering step, and perform FCM afterwards. The averaging and median filters, followed by FCM clustering, are referred to as FCM_S1 and FCM_S2, respectively 4 . Paper 11 also pointed out, that once having the neighbors evaluated, and thus for each pixel having extracted a scalar feature, FCM can be performed on the basis of the gray level histogram, clustering the gray levels instead of the pixels, which significantly reduces the computational load, as the number of gray levels is generally smaller by orders of magnitude. This latter quick approach, combined with an averaging pre-filter, is referred to as enhanced FCM (EnFCM) 3[11. All BCFCM, FCM_S1, and EnFCM suffer from the presence of a parameter denoted by $\alpha$, which controls the strength of the averaging effect, balances between the original and averaged image, and whose ideal value unfortunately can be found only experimentally. Another drawback is the fact, that averaging and median filtering, besides eliminating salt-and-pepper noises, also blurs relevant edges. Due to these shortcomings, Cai et al. 3 introduced a new local similarity measure, combining spatial and gray level distances, and applied it as an alternative pre-filtering to EnFCM, having this approach named fast generalized FCM (FGFCM). This approach is able to extract local information causing less blur than the averaging or median filter, but still has an experimentally adjusted parameter $\lambda_{g}$, which controls the effect of gray level differences.

In this paper we propose a novel method for MR brain image segmentation that simultaneously aims at high accuracy in image segmentation, low noise sensitivity, and high processing speed.

\section{Methods}

\subsection{Standard Fuzzy C-Means Algorithm}

The fuzzy c-means algorithm has successful applications in a wide variety of clustering problems. The traditional FCM partitions a set of object data into a number of $c$ clusters based on the minimization of a quadratic objective function. 
When applied to segment gray level images, FCM clusters the intensity level of all pixels $\left(x_{k}, k=1 \ldots n\right)$, which are scalar values. The objective function to be minimized is:

$$
J_{\mathrm{FCM}}=\sum_{i=1}^{c} \sum_{k=1}^{n} u_{i k}^{m}\left(x_{k}-v_{i}\right)^{2},
$$

where $m>1$ is the fuzzyfication parameter, $v_{i}$ represents the prototype value of cluster $i, u_{i k} \in[0,1]$ is the fuzzy membership function showing the degree to which pixel $k$ belongs to cluster $i$. According to the definition of fuzzy sets, for any pixel $k$, we have $\sum_{i=1}^{c} u_{i k}=1$. The minimization of the objective function is reached by alternately applying the optimization of $J_{\mathrm{FCM}}$ over $\left\{u_{i k}\right\}$ with $v_{i}$ fixed, $i=1 \ldots c$, and the optimization of $J_{\mathrm{FCM}}$ over $\left\{v_{i}\right\}$ with $u_{i k}$ fixed, $i=1 \ldots c, k=1 \ldots n[6]$. During each cycle, the optimal values are computed from the zero gradient conditions, and obtained as follows:

$$
\begin{gathered}
u_{i k}^{\star}=\frac{\left(v_{i}-x_{k}\right)^{-2 /(m-1)}}{\sum_{j=1}^{c}\left(v_{j}-x_{k}\right)^{-2 /(m-1)}} \quad \forall i=1 \ldots c, \forall k=1 \ldots n, \\
v_{i}^{\star}=\frac{\sum_{k=1}^{n} u_{i k}^{m} x_{k}}{\sum_{k=1}^{n} u_{i k}^{m}} \quad \forall i=1 \ldots c .
\end{gathered}
$$

After adequate initialization of centroids $v_{i}$, (2) and (3) are applied alternately until the norm of the variation of vector $\mathbf{v}$ is less than a previously set small value $\varepsilon$. FCM has invaluable merits in making optimal clusters, but in image processing it has severe deficiencies. The most important one is the fact, that it fails to take into consideration the position of pixels, which is also relevant information in image segmentation. This drawback led to introduction of spatial constraints into fuzzy clustering.

\subsection{Fuzzy Clustering Using Spatial Constraints}

Ahmed et al. 1] proposed a modification to the objective function of FCM, in order to allow the labeling of a pixel to be influenced by its immediate neighbors. This neighboring effect acts like a regularizer that biases the solution to a piecewise homogeneous labeling [1]. The objective function of BCFCM is:

$$
J_{\mathrm{BCFCM}}=\sum_{i=1}^{c} \sum_{k=1}^{n}\left[u_{i k}^{m}\left(x_{k}-v_{i}\right)^{2}+\frac{\alpha}{n_{k}} \sum_{r \in N_{k}} u_{i k}^{m}\left(x_{r}-v_{i}\right)^{2}\right],
$$

where $x_{r}$ represents the gray level of pixels situated in the neighborhood $N_{k}$ of pixel $k$, and $n_{k}$ is the cardinality of $N_{k}$. The parameter $\alpha$ controls the intensity of the neighboring effect, and unfortunately its optimal value can be found only experimentally. Having the neighbors computed in every computation cycle, this iterative algorithm performs extremely slowly.

Chen and Zhang 4 reduced the time complexity of BCFCM, by previously computing the neighboring averaging term or replacing it by a median filtered 
term, calling these algorithms FCM_S1 and FCM_S2, respectively. These algorithms outperformed BCFCM, at least from the point of view of time complexity.

Szilágyi et al. 11 proposed a regrouping of the processing steps of BCFCM. In their approach, an averaging filter is applied first, similarly to the neighboring effect of Ahmed et al. [1]:

$$
\xi_{k}=\frac{1}{1+\alpha}\left(x_{k}+\frac{\alpha}{n_{k}} \sum_{r \in N_{k}} x_{r}\right) .
$$

This filtering is followed by an accelerated version of FCM clustering. The acceleration is based on the idea, that the number of gray levels is generally much smaller than the number of pixels. In this order, the histogram of the filtered image is computed, and not the pixels, but the gray levels are clustered [1], by minimizing the following objective function:

$$
J_{\mathrm{EnFCM}}=\sum_{i=1}^{c} \sum_{l=1}^{q} h_{l} u_{i l}^{m}\left(l-v_{i}\right)^{2},
$$

where $h_{l}$ denotes the number of pixels with gray level equaling $l$, and $q$ is the number of gray levels. The optimization formulas in this case will be:

$$
\begin{array}{cc}
u_{i l}^{\star}=\frac{\left(v_{i}-l\right)^{-2 /(m-1)}}{\sum_{j=1}^{c}\left(v_{j}-l\right)^{-2 /(m-1)}} \quad \forall i=1 \ldots c, \forall l=1 \ldots q, \\
v_{i}^{\star}=\frac{\sum_{l=1}^{q} h_{l} u_{i l}^{m} l}{\sum_{l=1}^{q} h_{l} u_{i l}^{m}} \quad \forall i=1 \ldots c .
\end{array}
$$

EnFCM drastically reduces the computation complexity of BCFCM and its relatives 311. If the averaging pre-filter is replaced by a median filter, the segmentation accuracy also improves significantly [312].

\subsection{Fuzzy Clustering Using Spatial and Gray Level Constraints}

Based on the disadvantages of the aforementioned methods, but inspired of their merits, Cai et al. [3] introduced a local (spatial and gray) similarity measure that they used to compute weighting coefficients for an averaging pre-filter. The filtered image is then subject to EnFCM-like histogram-based fast clustering. The similarity between pixels $k$ and $r$ is given by the following formula:

$$
S_{k r}=\left\{\begin{array}{ll}
s_{k r}^{(s)} \cdot s_{k r}^{(g)} & \text { if } r \in N_{k} \backslash\{k\} \\
0 & \text { if } r=k
\end{array} .\right.
$$

where $s_{k r}^{(s)}$ and $s_{k r}^{(g)}$ are the spatial and gray level components, respectively. The spatial term $s_{k r}^{(s)}$ is defined as the $L_{\infty}$-norm of the distance between pixels $k$ and $r$. The gray level term is computed as $s_{k r}^{(g)}=\exp \left(-\left(x_{k}-x_{r}\right)^{2} /\left(\lambda_{g} \sigma_{k}^{2}\right)\right)$, where $\sigma_{k}$ denotes the average quadratic gray level distance between pixel $k$ and its neighbors. Segmentation results are reported more accurate than in any previously presented case 3 . 


\subsection{The Proposed Method}

Probably the most relevant problem of all techniques presented above, BCFCM, EnFCM, FCM_S1, and FGFCM, is the fact that they depend on at least one parameter, whose value has to be found experimentally. The parameter $\alpha$ balances the effect of neighboring in case of the former three, while $\lambda_{g}$ controls the tradeoff between spatial and gray level components in FGFCM.

The zero value in the second row of (9) implies, that in FGFCM, the filtered gray level of any pixel is computed as a weighted average of its neighbors. Having renounced to the original intensity of the current pixel, even if it is a reliable, noise-free value, unavoidably produces some extra blur into the filtered image. Accurate segmentation requires this kind of effects to be minimized 9.

In the followings we propose a set of modifications to EnFCM/FGFCM, in order to improve the accuracy of segmentation, without renouncing to the speed of histogram-based clustering. In other words, we need to define a complex filter that can extract relevant feature information from the image while applied as a pre-filtering step, so that the filtered image can be clustered fast afterwards based on its histogram. The proposed method consists of the following steps:

1 . As we are looking for the filtered value of pixel $k$, we need to define a small square or diamond-shape neighborhood $N_{k}$ around it. Square windows of size $3 \times 3$ were used throughout this study.

2. We search for the minimum, maximum, and median gray value within the neighborhood $N_{k}$, and we denote them by $\min _{k}, \max _{k}$ and $\operatorname{med}_{k}$, respectively.

3 . We replace the gray level of the maximum and minimum valued pixel with the median value (if there are more than one maxima or minima, replace them all), unless they are situated in the middle pixel $k$. In this latter case, pixel $k$ remains unchanged, just labeled as unreliable value.

4. Compute the average quadratic gray level difference of the pixels within the neighborhood $N_{k}$, using the formula

$$
\sigma_{k}=\sqrt{\frac{\sum_{r \in N_{k} \backslash\{k\}}\left(x_{r}-x_{k}\right)^{2}}{n_{k}-1}} .
$$

5. The filter coefficients will be defined as:

$$
C_{k r}=\left\{\begin{array}{ll}
c_{k r}^{(s)} \cdot c_{k r}^{(g)} & \text { if } r \in N_{k} \backslash\{k\} \\
1 & \text { if } r=k \wedge x_{k} \notin\left\{\max _{k}, \min _{k}\right\} \\
0 & \text { if } r=k \wedge x_{k} \in\left\{\max _{k}, \min _{k}\right\}
\end{array} .\right.
$$

The central pixel $k$ will be ignored if its value was found unreliable, otherwise it gets unitary weight. All other neighbor pixels will have coefficients $C_{k r} \in[0,1]$, depending on their space distance and gray level difference from the central pixel. In case of both terms, higher distance values push the coefficients towards 0 .

6 . The spatial component $c_{k r}^{(s)}$ is a negative exponential of the Euclidean distance between the two pixels $k$ and $r: c_{k r}^{(s)}=\exp \left(-L_{2}(k, r)\right)$. The gray level term depends on the difference $\left|x_{r}-x_{k}\right|$, according to a bell-shaped function defined as follows: 


$$
c_{k r}^{(g)}=\left\{\begin{array}{ll}
{\left[\cos \left(\pi \frac{x_{r}-x_{k}}{8 \sigma_{k}}\right)\right]^{2}} & \text { if }\left|x_{r}-x_{k}\right| \leq 4 \sigma_{k} \\
0 & \text { if }\left|x_{r}-x_{k}\right|>4 \sigma_{k}
\end{array} .\right.
$$

7. The extracted feature value for pixel $k$, representing its filtered intensity value, is obtained as a weighted average of its neighbors:

$$
\xi_{k}=\frac{\sum_{r \in N_{k}} C_{k r} x_{r}}{\sum_{r \in N_{k}} C_{k r}} .
$$

Algorithm. We can summarize the proposed method as follows:

1. Pre-filtering step: for each pixel $k$ of the input image, compute the filtered gray level value $\xi_{k}$, using (10), (11), (12), and (13).

2. Compute the histogram $h_{l}$ of the pre-filtered image, $l=1 \ldots q$.

3. Initialize $v_{i}$ with valid gray level values, differing from each other.

4. Compute new fuzzy memberships $u_{i l}, i=1 \ldots c, l=1 \ldots q$, using (7).

5. Compute new cluster prototypes $v_{i}, i=1 \ldots c$, using (8).

6. If there is relevant change in the $v_{i}$ values, go back to step 4 . This is tested by comparing any norm of the difference between the new and the old vector $\mathbf{v}$ with a preset small constant $\varepsilon$.

The algorithm converges quickly, however, the number of necessary iterations depends on $\varepsilon$ and on the initial cluster prototype values.

\section{Results and Discussion}

In this section we test and compare the accuracy of four algorithms: BCFCM, EnFCM, FGFCM, and the proposed method, on several synthetic and real images. All the following experiments used $3 \times 3$ window size for all kinds of filtering.

The proposed filtering technique uses a convolution mask whose coefficients are context dependent, and thus computed for the neighborhood of each pixel. Figure 1 presents the obtained coefficients for two particular cases. Figure 1(a) shows the case, when the central pixel is not significantly noisy, but some pixels in the neighborhood might be noisy or might belong to a different cluster. Under such circumstances, the upper three pixels having distant gray level compared to the value of the central pixel, receive small weights and this way they hardly contribute to the filtered value. Figure1(b) presents the case of an isolated noisy pixel situated in the middle of a relatively homogeneous window. Even though all computed coefficients are low, the noise is eliminated, resulting a convenient filtered value 111 . The migration of weights from the local maximum and minimum towards the median valued pixel, indicated by the arrows, is relevant in the second case and useful in the first.

The noise removal performances were compared using a $256 \times 256$-pixel synthetic test image taken from IBSR [7, having a high degree of mixed noise. Results are summarized in Fig. 2. Visually, the proposed method achieves best results, slightly over FGFCM, and significantly over all others. 


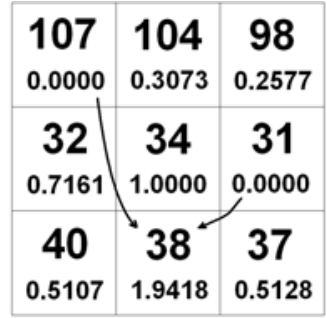

(a)

\begin{tabular}{|c|c|c|}
\hline 111 & 107 & 102 \\
0.0645 & 0.0668 & 0.0000 \\
\hline 120 & 204 & 108 \\
0.1523 & 0.0000 & 0.0723 \\
\hline 109 & 105 & 110 \\
\hline 0.1118 & 0.0564 & 0.0601 \\
\hline
\end{tabular}

(b)

Fig. 1. Filter mask coefficients in case of a reliable pixel intensity value (a), and a noisy one (b). The upper number in each cell is the intensity value, while the lower number represents the obtained coefficient. The arrows show, that the coefficients of extreme intensities are transferred to the median valued pixel.

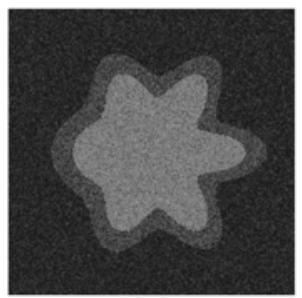

(a)

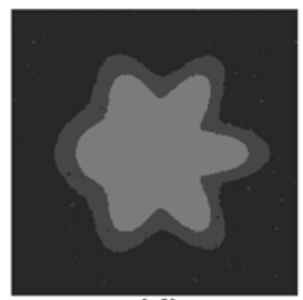

(d)

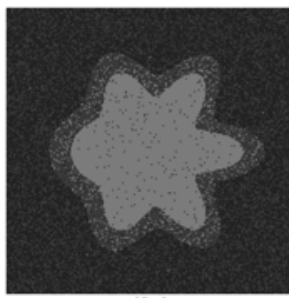

(b)

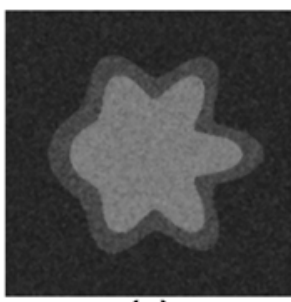

(e)

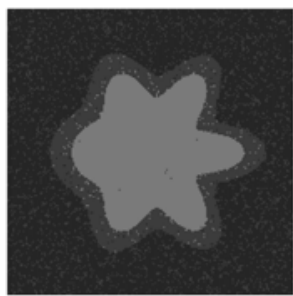

(c)

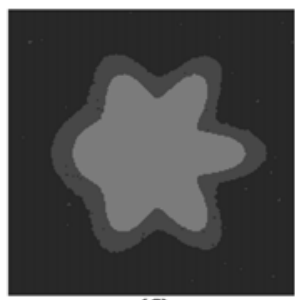

(f)

Fig. 2. Segmentation results on phantom images: (a) original, (b) segmented with traditional FCM, (c) segmented using BCFCM, (d) segmented using FGFCM, (e) filtered using the proposed pre-filtering, (f) result of the proposed segmentation

Table 1. Segmentation results on synthetic test images: misclassification rates at different noise types and levels

\begin{tabular}{llllr}
\hline Noise type & BCFCM & EnFCM & FGFCM & Proposed \\
\hline Gaussian 1 & $0.232 \%$ & $0.192 \%$ & $0.102 \%$ & $0.090 \%$ \\
Gaussian 2 & $7.597 \%$ & $0.972 \%$ & $0.405 \%$ & $0.330 \%$ \\
Gaussian 3 & $18.545 \%$ & $4.647 \%$ & $2.975 \%$ & $2.155 \%$ \\
Impulse & $0.250 \%$ & $0.192 \%$ & $0.130 \%$ & $0.120 \%$ \\
Mixed 1 & $0.345 \%$ & $0.220 \%$ & $0.130 \%$ & $0.110 \%$ \\
Mixed 2 & $5.542 \%$ & $1.025 \%$ & $0.675 \%$ & $0.547 \%$ \\
\hline
\end{tabular}


Table 1 gives a statistical analysis of the synthetic images contaminated with different noises (Gaussian noise, salt-and-pepper impulse noise, and mixtures of these) at different levels. The table reveals that the proposed filter performs best at removing all these kinds of noises. Consequently, the proposed method is suitable for segmenting images corrupted with unknown noises, and in all cases it performs at least as well as his ancestors.

We applied the presented filtering and segmentation techniques to several T1weighted real MR images. A detailed view, containing numerous segmentations, is presented in Fig. 3

The original slice in Fig. 3(a) is taken from IBSR. We produced several noisy versions of this slice, by adding salt-and-pepper impulse noise and/or Gaussian

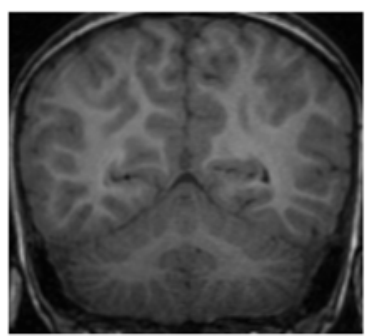

(a)

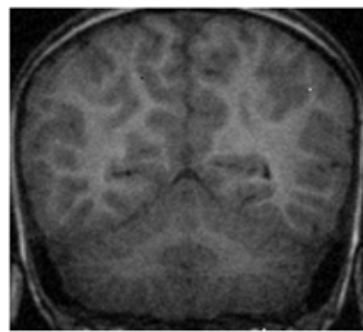

(d)

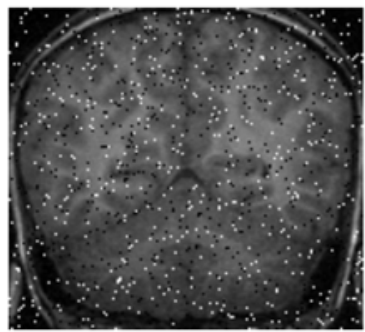

(g)

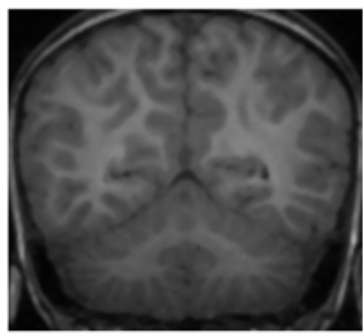

(b)

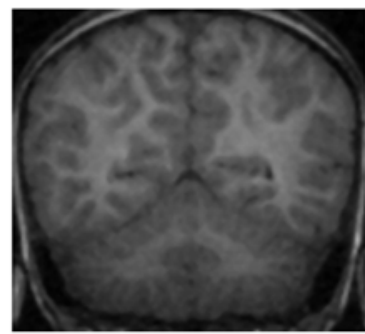

(e)

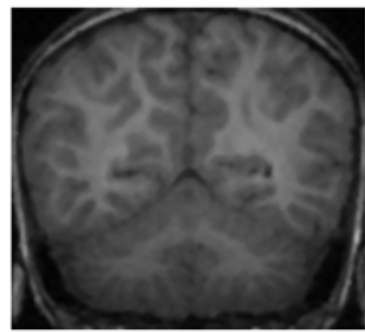

(h)

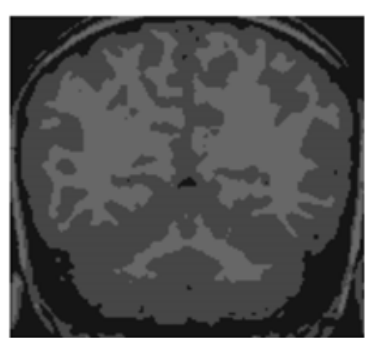

(c)

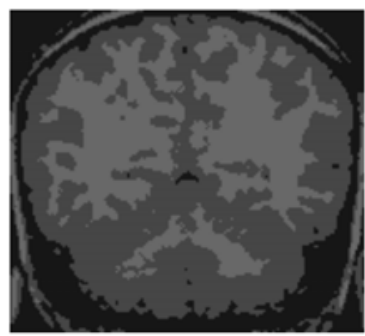

(f)

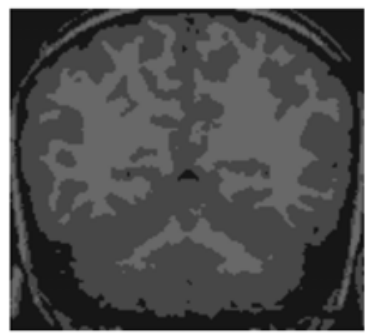

(i)

Fig. 3. Filtering and segmentation results on real T1-weighted MR brain images, corrupted with different kinds and levels of artificial noise. Each row contains an original or noise-corrupted brain slice on the left side, the filtered version (using the proposed method) in the middle, and the segmented version on the right side. Row (a)-(c) comes from record number 1320_2_43 of IBSR [7], row (d)-(f) is corrupted with $10 \%$ Gaussian noise, while row (g)-(i) contains mixed noise of $5 \%$ impulse $+5 \%$ Gaussian. 
noise, at different intensities. Some of these noisy versions are visible in Fig. 3 (d), (g). The filtered versions of the three above mentioned slices are presented in the middle column of Fig. 3. The segmentation results of the chosen slices are shown in Fig. 3 (c), (f), (i). From the segmented images we can conclude, that the proposed filtering technique is efficient enough to make proper segmentation of any likely-to-be-real MRI images in clinical practice, at least from the point of view of Gaussian and impulse noises.

Table 2 takes into account the behavior of three mentioned segmentation techniques, in case of different noise types and intensities, computed by averaging the misclassifications on 12 different T1-weighted real MR brain slices, all taken from IBSR. The proposed algorithm has lowest misclassification rates in most of the cases.

Table 2. Misclassification rates in case of real brain MR image segmentation

\begin{tabular}{lccr}
\hline Noise type & EnFCM & FGFCM & Proposed \\
\hline Original, no extra noise & $0.767 \%$ & $0.685 \%$ & $0.685 \%$ \\
Gaussian 4\% & $1.324 \%$ & $1.131 \%$ & $1.080 \%$ \\
Gaussian 8\% & $3.180 \%$ & $2.518 \%$ & $1.489 \%$ \\
Gaussian 12\% & $4.701 \%$ & $2.983 \%$ & $2.654 \%$ \\
Impulse 1\% & $0.836 \%$ & $0.717 \%$ & $0.726 \%$ \\
Impulse 3\% & $1.383 \%$ & $0.864 \%$ & $0.823 \%$ \\
Impulse 5\% & $1.916 \%$ & $1.227 \%$ & $0.942 \%$ \\
Impulse 10\% & $3.782 \%$ & $1.268 \%$ & $1.002 \%$ \\
Impulse 5\%+ Gaussian 4\% & $2.560 \%$ & $1.480 \%$ & $1.374 \%$ \\
Impulse 5\%+ Gaussian 8\% & $3.626 \%$ & $2.013 \%$ & $1.967 \%$ \\
Impulse 5\%+ Gaussian 12\% & $6.650 \%$ & $4.219 \%$ & $4.150 \%$ \\
\hline
\end{tabular}

Further tests also revealed, that the proposed method performs well in case of T2-weighted MR brain images, too. We applied the proposed segmentation method to several complete head MR scans in IBSR. The dimensions of the image stacks were $256 \times 256 \times 64$ voxels. The average total processing time for one stack was around 10 seconds on a $2.4 \mathrm{GHz}$ Pentium 4.

\section{Conclusions}

We have developed a modified FCM algorithm for automatic segmentation of MR brain images. The algorithm was presented as a combination of a complex pre-filtering technique and an accelerated FCM clustering performed over the histogram of the filtered image. The pre-filter uses both spatial and gray level criteria, in order to achieve efficient removal of Gaussian and impulse noises without significantly blurring the real edges. Experiments with synthetic phantoms and real MR images show, that our proposed technique accurately segments the different tissue classes under serious noise contamination. We compared our 
results with other recently reported methods. Test results revealed that our approach performed better than these methods in many aspects, especially in the accuracy of segmentation and processing time.

Although the proposed method segments 2-D MR brain slices, it gives a relevant contribution to the accurate volumetric segmentation of the brain, because the segmented images and the obtained fuzzy memberships can serve as excellent input data to any level set method that constructs $3-\mathrm{D}$ cortical surfaces.

Further works aim to reduce the sensitivity of the proposed technique to intensity inhomogeneity noises, and to introduce adaptive determination of the optimal number of clusters.

Acknowledgements. This research was supported by the Sapientia Institute for Research Programmes, Domus Hungarica Scientiarium et Artium, the Communitas Foundation, and the Pro Progressio Foundation.

\section{References}

1. Ahmed, M.N., Yamany, S.M., Mohamed, N., Farag, A.A., Moriarty, T.: A modified fuzzy c-means algorithm for bias field estimation and segmentation of MRI data. IEEE Trans. Med. Imag. 21, 193-199 (2002)

2. Bezdek, J.C., Pal, S.K.: Fuzzy models for pattern recognition. IEEE Press, Piscataway, NJ (1991)

3. Cai, W., Chen, S., Zhang, D.Q.: Fast and robust fuzzy c-means algorithms incorporating local information for image segmentation. Patt. Recogn. 40, 825-838 (2007)

4. Chen, S., Zhang, D.Q.: Robust image segmentation using FCM with spatial constraints based on new kernel-induced distance measure. IEEE Trans. Syst. Man. Cybern. Part. B. 34, 1907-1916 (2004)

5. Chuang, K.S., Tzeng, H.L., Chen, S., Wu, J., Chen, T.J.: Fuzzy c-means clustering with spatial information for image segmentation. Comp. Med. Imag. Graph. 30, 9-15 (2006)

6. Hathaway, R.J., Bezdek, J.C., Hu, Y.: Generalized fuzzy c-means clustering strategies using $L_{p}$ norm distances. IEEE Trans. Fuzzy Syst. 8, 576-582 (2000)

7. Internet Brain Segmentation Repository, at http://www.cma.mgh.harvard.edu/ ibsr

8. Pham, D.L., Prince, J.L.: Adaptive fuzzy segmentation of magnetic resonance images. IEEE Trans. Med. Imag. 18, 737-752 (1999)

9. Pham, D.L.: Unsupervised tissue classification in medical images using edgeadaptive clustering. Proc. Ann. Int. Conf. IEEE EMBS 25, 634-637 (2003)

10. Siyal, M.Y., Yu, L.: An intelligent modified fuzzy c-means based algorithm for bias field estimation and segmentation of brain MRI. Patt. Recogn. Lett. 26, 2052-2062 (2005)

11. Szilágyi, L., Benyó, Z., Szilágyi, S.M., Adam, H.S.: MR brain image segmentation using an enhanced fuzzy C-means algorithm. Proc. Ann. Int. Conf. IEEE EMBS 25, 724-726 (2003)

12. Szilágyi, L.: Medical image processing methods for the development of a virtual endoscope. Period. Polytech. Ser. Electr. Eng. 50(1-2), 69-78 (2006) 